\title{
Genuine Palestinian cabbage: Myth or fact. Comparison between Palestinian and Belgian coronary artery bypass grafting
}

\author{
Basma Salameh $^{1 *}$, Ahmad K. Darwazah ${ }^{2}$, Henri Verhaaren ${ }^{3}$, Guido Van Nooten ${ }^{4}$ \\ ${ }^{1}$ Doctoral School, Department of Medical-Social Science, Ghent University, Ghent, Belgium \\ ${ }^{2}$ Head of Department of Cardiac Surgery, Ramallah Hospital, Consultant Cardiac Surgeon, Makassed Hospital, Jerusalem, Palestine \\ ${ }^{3}$ Ghent University, Ghent, Belgium \\ ${ }^{4}$ Department of Cardiac Surgery, University Hospital Ghent, Ghent, Belgium \\ Email: ${ }^{\text {basmas.salameh@ugent.be }}$
}

Received 3 April 2013; revised 3 May 2013; accepted 16 May 2013

Copyright (C) 2013 Basma Salameh et al. This is an open access article distributed under the Creative Commons Attribution License, which permits unrestricted use, distribution, and reproduction in any medium, provided the original work is properly cited.

\section{ABSTRACT}

Background: Outcomes post-CABG serves as an indicator of the quality and effectiveness of care given by health care providers. The primary aim of this study is to describe outcomes such as mortality, morbidity, length of hospitalization and hospital readmission post-CABG between Ramallah Ministry of Health Hospital and Ghent University hospital. Methods: A retrospective study is conducted on 200 matched patients who underwent isolated CABG in Ramallah hospital and Ghent University hospital between 2009 and 2011. Both groups were followed up in the post-operative period for minimum one year. Inferential statistics were performed to find the presence of relationship among variables. Kaplan-Meyer was performed to compare overall survival rates and freedom from adverse events. The logistic model was used to assess all factors that associated with readmission, mortality, morbidity and hospital stay. Findings: Both groups had comparable patient characteristics and operative data. Risk factors of diabetes $(50 \%)$ and smoking $(60 \%)$ were higher in Palestinian patients compared to $(29 \%)$ and $(31 \%)$ in Belgian patients. The mortality rate slightly, being higher among the Palestinian group, was $4 \%$ compared to $2 \%$ among the Belgium group. Occurrence of morbidity was less noted among Palestinian patients compared to Belgium patients $(23 \%, 36 \%, P=0.044$ respectively). Palestinian patients had shorter hospital stay (IQR = $6-10, P<0.001)$. However, they had significantly higher rate of re-admission within 30 days of the operation $(P=0.007)$. Moreover, the overall in-

\footnotetext{
"Corresponding author.
}

fection experience in the Palestinian group was higher after adjustment for the confounding effect of smoking and diabetes $(P=0.046$ and $P=0.042$ respectively). In Ramallah hospital, diabetes $(P=0.011)$ and smoking $(P=0.012)$ were significantly associated with higher readmission rate. Interpretation: Finding indicates that although in-hospital adverse events didn't statistically differ, 30-day mortality rate and re-admission were slightly higher among Palestinian patients, while the incidence of atrial fibrillation was increased and hospital stay was prolonged in the Belgium group. Diabetes and smoking in Palestine were found to be independent risk factors for higher readmission rate and infection.

Keywords: CABG; Mortality; Morbidity; Length of Hospitalization; Re-Admission; Atrial Fibrillation; Palestine

\section{INTRODUCTION}

Coronary Artery Disease (CAD) is a leading health problem worldwide. It is a major cause of disability and death and is responsible for roughly 20 percent of the mortality in the western world [1], where recently a decline in mortality has been observed in developed countries, both CAD mortality and risk factors are on the rise in developing countries [2-4].

Management of CAD with surgical revascularization by coronary artery bypass grafting (CABG) is recommended for patients with severe blockage more than $70 \%$ in the left main and the proximal left anterior descending coronary artery, multi-vessel disease, or disabling angina refractory to medical treatment [5]. CABG is indicated to 
relieve symptoms, attain better health, improve quality of life (QoL), improve survival and/or prolong life and not to cure the disease $[6,7]$.

In Palestinian territories, the main leading cause of death is cardiovascular disease and represents 22.4 percent of the total deaths in 2011 [8]. However, published studies on the coronary care system are mainly based on data from East-Jerusalem Arab and Jewish citizens [9, 10]. The Palestinian Ministry of Health $(\mathrm{MoH})$ made a priority of the empowerment of intensive cardiac facilities and manpower in West Bank and Gaza, as reflected in the National Health strategic plans [11]. In 1999, the first CABG was successfully performed in MoH Ramallah hospital in the West Ban.

This study compares the data between two institutions of patients who underwent isolated CABG: from Occupied Palestinian territories being operated at MoH Ramallah hospital and from Belgium being operated at Ghent University hospital.

The specific aims of this study were:

1) To compare in-hospital mortality and morbidity post isolated CABG for both groups. The dependent variables were 30-day mortality, post-operative complications and length of hospitalization.

2) To compare out-hospital mortality and morbidity post isolated CABG for both groups. The dependent variables were 30 -day mortality, overall mortality, postoperative complications, 30-day readmission rates and length of hospitalization.

3) To investigate the predictors of mortality, morbidity, length of hospitalization and readmission rate for both groups.

\section{MATERIALS AND METHODS}

\subsection{Study Population}

Clinical data were collected retrospectively and selected from 100 patients from Palestine and matched with 100 patients from Belgium who underwent On-pump or Offpump isolated coronary artery bypass grafting (CABG) and were operated between 2009 and 2011. All cases were matched in terms of age and sex. Patients were excluded from the study if they had other cardiac surgery than isolated CABG during the surgery. Risk stratification was performed using the EuroSCORE model and obtained through history, investigations and review of the medical records in the preoperative period. Post-operative data were also collected and included 30-days mortality rates, length of hospitalization, readmission rates, and post-operative complications: including bleeding, infection, atrial fibrillation, ventricular arrhythmias, CVA, myocardial infarction, respiratory failure and renal failure. Following this retrospective data collection, the patients were followed up in the post-operative period for minimum one year. Readmission after discharge was only counted when the adverse event was related to CABG surgery and the other reasons were excluded. It is important to note that nine female patients from the Palestinian group were excluded from the study because of their young age $(<45$ years) since they couldn't be matched by female Belgian patients of that age. These patients were substituted with other matched yet elderly cases.

\subsection{Ethical Consideration}

Ethical approval was obtained from the ethical committee of Ramallah hospital as well as from Helsinki Committee, Palestine. Also support was obtained from the Chief of Cardiothoracic Surgery unit from both hospitals. Once the data was collected all patients were assigned a code number and patient's original names were only accessible to the researcher.

\subsection{Operational Definitions of Study Variables}

Mortality: death from any cause related to CABG surgery within 30 days of operation and within the follow up period.

Length of hospitalization: total days spent in the hospital from admission to discharge. The dates were noted on patient profiles.

Readmission: number of patients readmitted to the health institution post cardiac surgery for complications related to CABG surgery within 30 days of operation and during follow up period.

Outcomes: adverse events in terms of: mortality; morbidity (bleeding, infection, atrial fibrillation, ventricular arrhythmias, CVA, myocardial infarction, respiratory failure and renal failure); hospital readmission rates.

\subsection{EuroSCORE}

The European System for Cardiac Operative Risk Evaluation (EuroSCORE) is a valuable tool to predict mortality post cardiac surgery. Two models have been developed EuroSCORE I published in 1999 and a new readjusted model EuroSCORE II launched in 2011 $[12,13]$. EuroSCORE I was used to evaluate the risk variables of the pre-operative clinical variables of the patients in both groups: age, gender, left ventricular ejection fraction, serum creatinin $>200 \mu \mathrm{mol} / \mathrm{L}$, cardiac arteriopathy urgent surgery, COPD, critical preoperative state, unstable angina and recent myocardial infarction $<90$ days, and not to validate it as a predictive model of mortality.

Two risk evaluation score of the EuroSCORE model are available - the simple additive EuroSCORE and the 
logistic EuroSCORE. For patients undergoing On-pump and Off-pump CABG, this model has demonstrated a quality indicator for prediction of postoperative death postcardiac surgery [14].

\subsection{Follow Up}

At the time of the follow-up, medical records of each patient were accessed using patient's unique medical record number and reviewed in terms of mortality, morbidity and re-admission. Collection of data during follow up phase for both hospitals was performed over a period of six weeks. In Belgium, most patients were reviewed at the University hospital. If patients were followed in another hospital data were collected from the family physician. The situation in Palestine was completely different, due to a lack of information for some patients who went to surgery during 1999 six new patients were substituted. Also, Ramallah hospital is the referral hospital in Palestine and provides its services for patients from different cities. Most of these patients are followed in the clinics where they live and some of them simply skip follow-up. For that subset of participants, structured phone interviews were carried out by the researcher.

\subsection{Statistical Analysis}

Data entry and statistical analysis were done using SPSS 20.0 statistical software package Chicago, Ill, USA). The selection of inferential statistics for between- and withingroup comparison was dependent on the measurement level of the data as well as the result of the normality and homogeneity of variance tests. For those data which are normally distributed and the homogeneity of variance test is insignificant, parametric inferential statistics were applied. Otherwise, non-parametric tests were used. Quantitative continuous data are presented as mean \pm SD and were compared using two tailed Student $t$-test and Mann-Whitney U-test in case of comparisons between two groups. Qualitative categorical variables are provided as frequencies and percentages and were compared using chi-square test. Fisher exact test was employed in all cases when the expected value in the $2 \times 2$ table is less than 5. Kaplan-Meyer Survival analysis was performed and compared between groups using the log-rank and Wilcoxon tests. A two tailed statistical significance was considered at a tow-tailed $\mathrm{P}$-value $<0.05$. Univariate analysis was done and when the P-value $<0.05$ the variables was included in logistic regression using backward stepwise. The logistic multivariate model was used to ascertain all relevant factors that were associated with readmission, mortality, morbidity and length of hospitalization. The model's goodness-of-fit was evaluated using Hosmer-Lemeshow statistic.

\section{RESULTS}

\subsection{Matching Characteristics}

Table 1 confirms that patients from Palestine and Belgium had matching characteristics; almost the same mean age $(59.0 \pm 7.5$ (range $=40-76$ years) and $59.2 \pm$ 8.0 (range $=41-79$ years), respectively and for both groups, $18 \%$ of the subjects were female. Operation techniques were similar. Sixty three percent of the patients were operated using the On-pump technique and $37 \%$ were operated using the Off-pump technique. There was no significant difference in the number of distal anastomoses.

\subsection{Risk Factors}

Table 2 shows that both groups had similar documented hypertension and dyslipidemia. Overweight status was slightly higher in Palestinian patients (56\% compared to $45 \%$ for Belgian patients). Palestinian patients had significantly less positive family history of atherosclerosis $7 \%$, but more history of DM $50 \%$ and smoking $60 \%$, compared to the $40 \%, 29 \%$ and $31 \%$ in Belgian patients.

\subsection{Clinical Preoperative Data}

In terms of pre-operative data, Table 3 shows that $46 \%$ Palestinian patients had significantly more medium $(30 \%$ - 50\%) left ventricular ejection fraction(LVEF) and less good LVEF ( $>50 \%$ ) compared to $8 \%$ and $84 \%$, respectively to patients in the Belgian group. Belgium patients

Table 1. Operative data of patients in the two study groups.

\begin{tabular}{|c|c|c|c|}
\hline & \multicolumn{2}{|c|}{ Group } & \multirow{2}{*}{ P-value } \\
\hline & $\begin{array}{l}\text { Palestine } \\
(\mathrm{n}=100)\end{array}$ & $\begin{array}{l}\text { Belgium } \\
(\mathrm{n}=100)\end{array}$ & \\
\hline Age $($ mean $\pm \mathrm{SD})$ : & $59.0 \pm 7 \cdot 5$ & $59.2 \pm 8.0$ & \multirow{2}{*}{0.827} \\
\hline Range: & $40.0-76.0$ & $41.0-79.0$ & \\
\hline \multicolumn{4}{|l|}{ Sex } \\
\hline Female & 18 & 18 & \multirow[t]{2}{*}{1.000} \\
\hline Male & 82 & 82 & \\
\hline \multicolumn{4}{|l|}{ Pump: } \\
\hline On Pump* & 63 & 63 & \\
\hline $\mathrm{OPCAB}^{\dagger}$ & 37 & 37 & 1.000 \\
\hline \multicolumn{4}{|c|}{ No. of distal anastomoses: } \\
\hline 1 & 7 & 4 & \\
\hline 2 & 32 & 32 & \\
\hline 3 & 52 & 51 & \\
\hline 4 & 9 & 13 & 0.666 \\
\hline
\end{tabular}


Table 2. Risk factors for both groups.

\begin{tabular}{lccc}
\hline & \multicolumn{2}{c}{ Group } & \\
\cline { 2 - 3 } & $\begin{array}{c}\text { Palestine } \\
(\mathrm{n}=100)\end{array}$ & $\begin{array}{c}\text { Belgium } \\
(\mathrm{n}=100)\end{array}$ & \\
\hline BMI: $^{*}$ & 21 & 28 & \\
$\quad$ Normal: $\geq 18.5$ & 56 & 45 & 0.284 \\
Overweight: $\geq 25$ & 23 & 27 & \\
Obese: $\geq 30$ & 7 & 40 & $<0.001$ \\
Family history & 50 & 29 & 0.002 \\
Diabetes mellitus & 66 & 71 & 0.447 \\
Hypertension & 70 & 78 & 0.197 \\
Dyslipidemia & 60 & 31 & $<0.001$ \\
Smoking & & &
\end{tabular}

*BMI: Body Mass Index.

have significantly more extra cardiac arteriopathy (25\%, $\leq 0.001)$. By contrast, Palestinian patients had more urgent surgery $(25 \%, \mathrm{P}=0.005)$, more COPD $(\mathrm{P}=0.036)$ and more recent $\mathrm{MI}(\mathrm{P} \leq 0.001)$ than patients in the Belgian group. Both groups had similar standard EuroSCOREs and logistic EuroSCOREs (Table 4).

\subsection{Post-Operative Data}

\subsubsection{Mortality}

Early mortality rate was slightly higher amongst the Palestinian group. As illustrated in Figure 1, the two groups did not differ in their overall mortality experience, with no statistically significant difference in mean survival days. However, the mortality rate of $4 \%$ within 30 days of the operation was significantly higher in Palestinian patients (log-rank $\mathrm{P}=0.044)$ when compared to Belgian patients.

\subsubsection{Mortality as Adjusted for Confounding Effect}

Overall mortality experience, in the Palestinian group, was not statistically significantly different from the Belgian group in mean survival even after adjustment for the possible confounding effect of LVEF, recent MI, and DM. However, it was statistically significant in the Belgium group and is shown by the result of the log-rank test $\mathrm{P}=0.004, \mathrm{P}=0.029$, and $\mathrm{P}=0.026$ respectively (Figures 2-4).

\subsubsection{Morbidity}

The two groups did not differ in their overall morbidity experience, with no statistically significant difference in the mean survival of days free from morbidity. Table 5 reports significantly less morbidity particularly atrial fibrillation for patients in the Palestinian group, compared to patients in the Belgian group $(23 \%, 36 \%$, respectively,

Table 3. Clinical pre-operative data of patients in the two study groups.

\begin{tabular}{ccc}
\hline \multicolumn{2}{c}{ Group } & \\
\cline { 2 - 3 } & $\begin{array}{c}\text { Palestine } \\
(\mathrm{n}=100)\end{array}$ & $\begin{array}{c}\text { Belgium } \\
(\mathrm{n}=100)\end{array}$ \\
\hline
\end{tabular}

Urgent:

No

Yes

$75 \quad 90$

25

0.005

$\mathrm{SC}>200 \mu \mathrm{mol} / \mathrm{L}^{\wedge}$ :

No

Yes

98

93

0.170

LVEF $^{\dagger}$ :

Good: $>50 \%$

Medium: $30 \%-50 \%$

Bad: $<30 \%$

10

COPD*:

No

Yes

Extra-cardiac arteriopathy:

$$
\text { No }
$$

Yes

Critical preoperative state:

No

Yes

Unstable angina:

$\begin{array}{lccc}\text { No } & 76 & 74 & 0.744 \\ \text { Yes } & 24 & 26 & \\ \text { Recent MI }{ }^{\#}(<90 \text { days }): & & & \\ \text { No } & 91 & 70 & <0.001 \\ \text { Yes } & 9 & 30 & \end{array}$

${ }^{\wedge} \mathrm{SC}$ : Serum Creatinin; ${ }^{*}$ COPD: Chronic Obstructive Pulmonary Disease; LVEF: Left ventricle ejection fraction; ${ }^{*}$ MI: myocardial Infarction.

Table 4. EuroScore.

\begin{tabular}{cccccc}
\hline & \multicolumn{4}{c}{ Group } & \\
\cline { 2 - 5 } & \multicolumn{2}{c}{$\begin{array}{c}\text { Palestine } \\
(\mathrm{n}=100)\end{array}$} & \multicolumn{2}{c}{$\begin{array}{c}\text { Belgium } \\
(\mathrm{n}=100)\end{array}$} & P-value \\
\cline { 2 - 5 } & Mean & SD & Mean & SD & \\
\hline EuroSCORE $(\log )$ & 0.05 & 0.072 & 0.049 & 0.077 & 0.896 \\
EuroSCORE & 3.7 & 3.3 & 3.9 & 3.3 & 0.485 \\
\hline
\end{tabular}




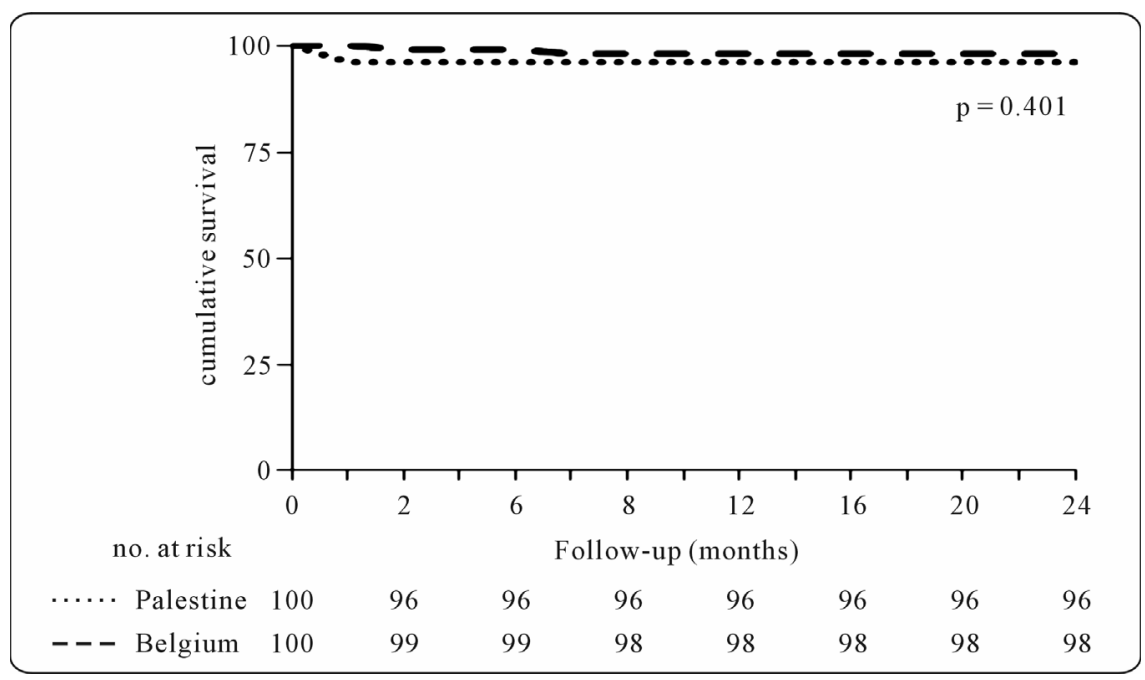

Figure 1. Mortality among patients in the two study groups.

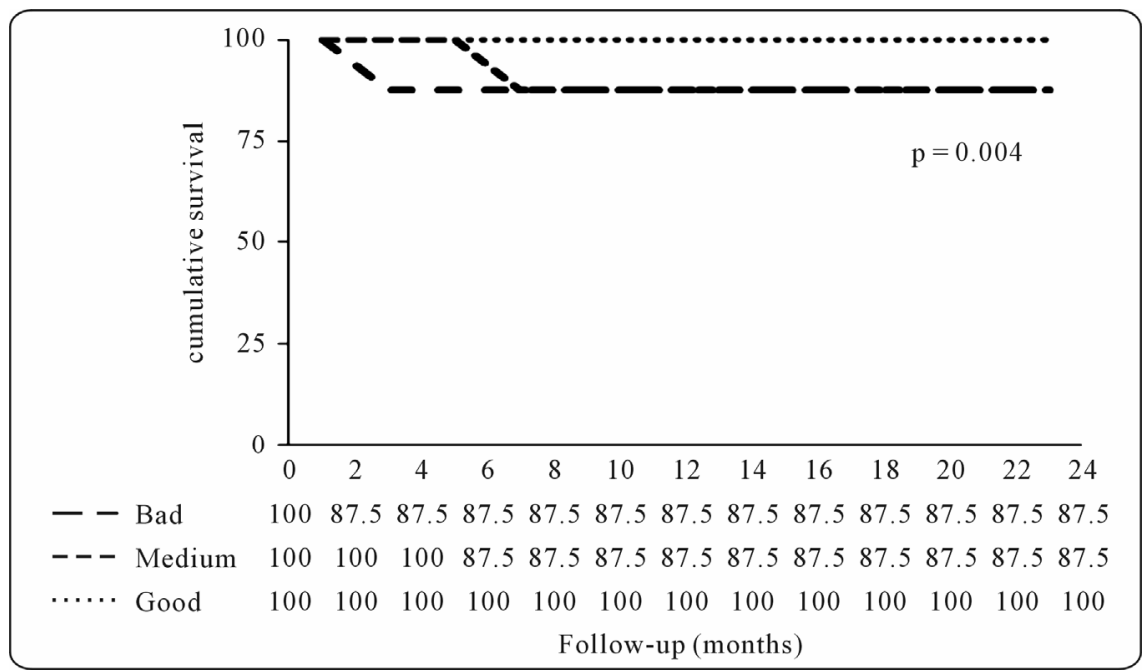

Figure 2. Overall mortality adjusted for LVEF among patients in Belgium.

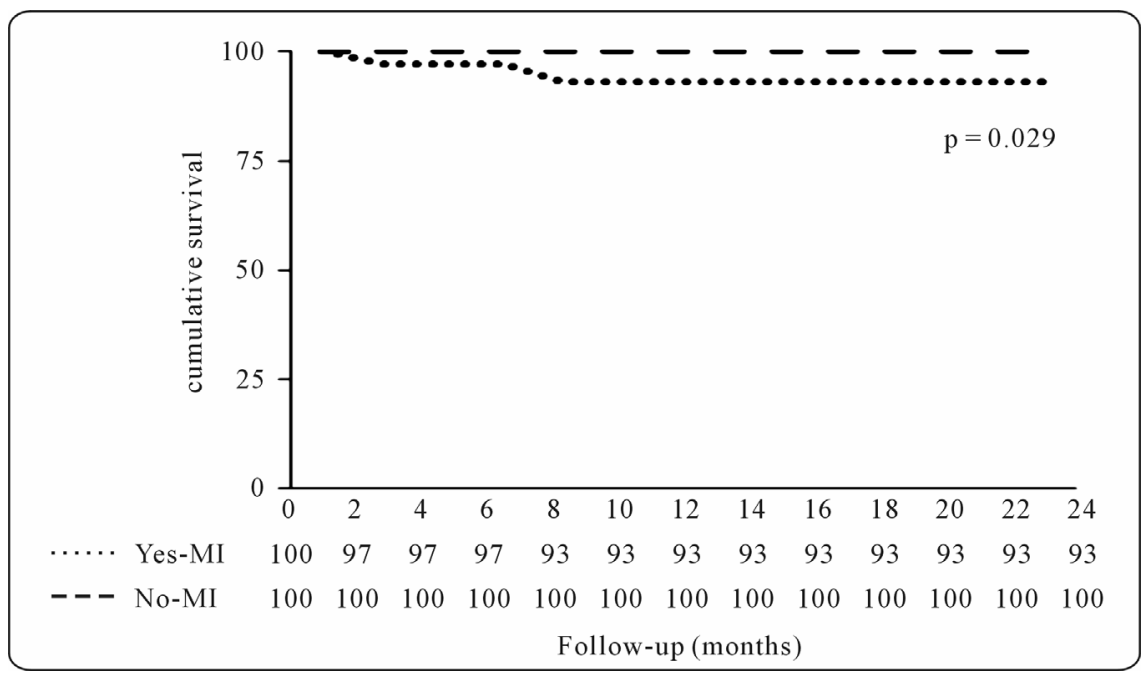

Figure 3. Overall mortality adjusted for recent myocardial infarction ( $<90$ days) among patients in Belgium. 


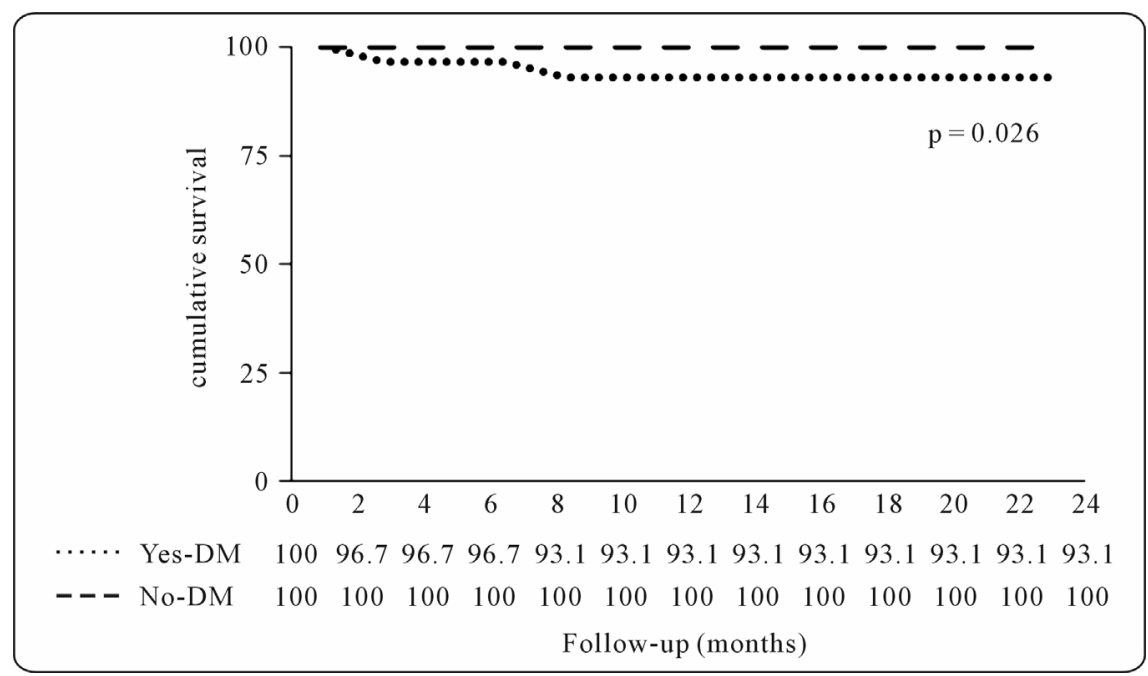

Figure 4. Overall mortality adjusted for Diabetes among patients in Belgium.

Table 5. Post-operative data for two groups.

\begin{tabular}{|c|c|c|c|}
\hline & \multicolumn{2}{|c|}{ Group } & \multirow{2}{*}{ P-value } \\
\hline & $\begin{array}{l}\text { Palestine } \\
(\mathrm{n}=100)\end{array}$ & $\begin{array}{l}\text { Belgium } \\
(\mathrm{n}=100)\end{array}$ & \\
\hline \multicolumn{4}{|l|}{ Mortality: } \\
\hline No & 96 & 98 & \\
\hline Yes & 4 & 2 & 0.683 \\
\hline \multicolumn{4}{|l|}{ Morbidity: } \\
\hline No & 77 & 64 & \\
\hline Yes & 23 & 36 & 0.044 \\
\hline In-hospital complications & 14 & 35 & $<0.001$ \\
\hline Bleeding & 5 & 7 & 0.552 \\
\hline Infection: & 10 & 9 & 0.809 \\
\hline Respiratory- infection & 4 & 7 & 0.352 \\
\hline Surgical-wound infection & 6 & 1 & 0.365 \\
\hline Catheter-UTI ${ }^{*}$ & 0 & 1 & 1.000 \\
\hline At Atrial fibrillation & 5 & 17 & 0.007 \\
\hline Ventricular arrhythmia & 2 & 1 & 1.000 \\
\hline Myocardial infarction & 2 & 0 & 0.497 \\
\hline $\mathrm{CVA}^{\#}$ & 2 & 2 & 1.000 \\
\hline Respiratory failure & 1 & 6 & 0.118 \\
\hline Renal failure & 2 & 6 & 0.279 \\
\hline Readmission & 10 & 3 & 0.045 \\
\hline One month Readmission & 8 & 0 & 0.007 \\
\hline
\end{tabular}

"UTI: Urinary Tract Infection; ${ }^{\#}$ CVA: Cerebrovascular Accident.

$P=0.044)$. However, they had significantly higher rate of re-admission $(\mathrm{P}=0.045)$ especially within 30 days of the operation $(\mathrm{P}=0.007)$. Palestinians (Table 6) had
Table 6. Follow-up days of patients in the two study groups.

\begin{tabular}{|c|c|c|c|c|c|}
\hline & \multicolumn{4}{|c|}{ Group } & \multirow{3}{*}{ P-value } \\
\hline & \multicolumn{2}{|c|}{$\begin{array}{l}\text { Palestine } \\
(\mathrm{n}=100)\end{array}$} & \multicolumn{2}{|c|}{$\begin{array}{c}\text { Belgium } \\
(\mathrm{n}=100)\end{array}$} & \\
\hline & Mean & SD & Mean & SD & \\
\hline LOS (days) ${ }^{*}$ & 8.9 & & 11.5 & & \\
\hline IQR (25 - 75) & $6-10$ & 6.7 & $9-11$ & 6.1 & $<0.001$ \\
\hline Rang & $2-63$ & & $7-47$ & & \\
\hline Postoperative Stay & 8.0 & 6.7 & 10.4 & 6.8 & $<0.001$ \\
\hline Follow-up (days) & 490.3 & 160.8 & 504.6 & 148.5 & 0.744 \\
\hline Follow-up (months) & 16.3 & 5.4 & 16.67 & 4.84 & 0.972 \\
\hline
\end{tabular}

significantly less postoperative days and lower hospital lengths of stay $(\mathrm{P} \leq 0.001)$.

\subsubsection{Morbidity as Adjusted for Confounding Effect} As displayed in Figures 5-7 overall morbidity experience in the Palestinian group was statistically significantly different from the Belgian group in mean survival days after adjustment for the possible confounding effect of unstable angina (log-rank test: $\mathrm{P}=0.042$ ), critical preoperative state (log-rank test: $\mathrm{P}=0.033$ ) and recent MI (log-rank test: $\mathrm{P}=0.009$ ). Moreover, overall infection experience in the Palestine group was statistically significant higher in the mean survival of days after adjustment for the possible confounding effect of risk factors smoking and $\mathrm{DM}(\log$ rank test: $\mathrm{P}=0.046$ and $\mathrm{P}=0.042$ respectively) as shown in Figure 8, 9. However, in the Belgian group overall morbidity experience was statistically significantly higher after adjustment for the possible 


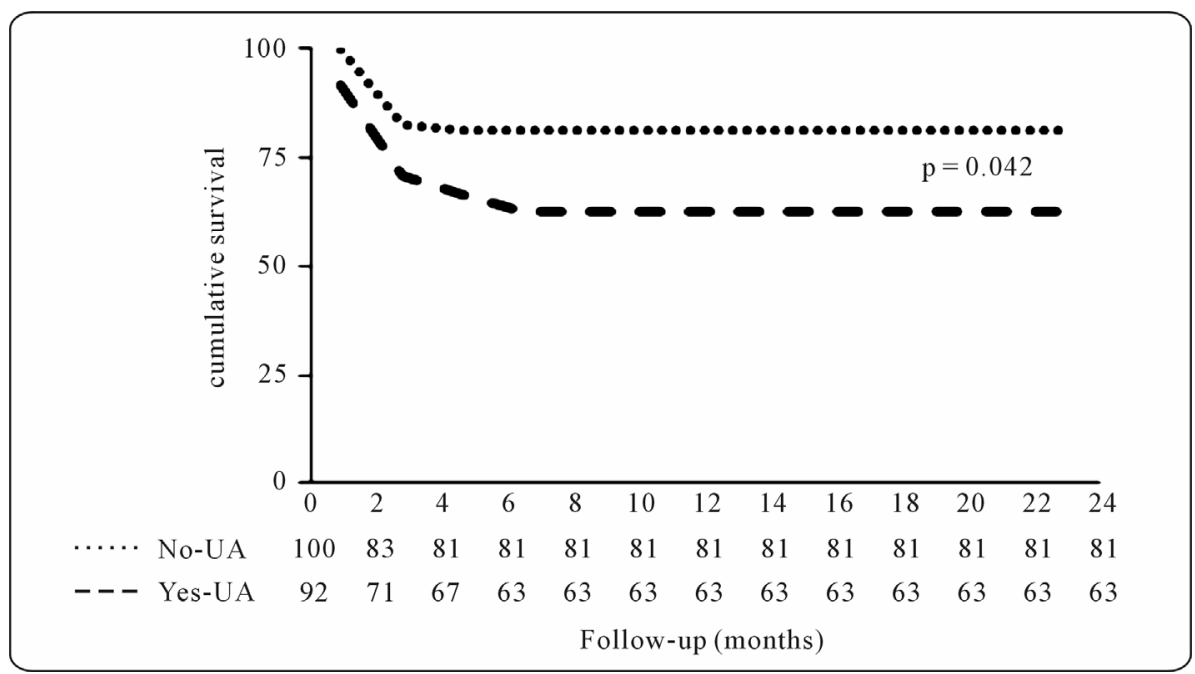

Figure 5. Overall morbidity adjusted for unstable angina (UA) among patients in Palestine.

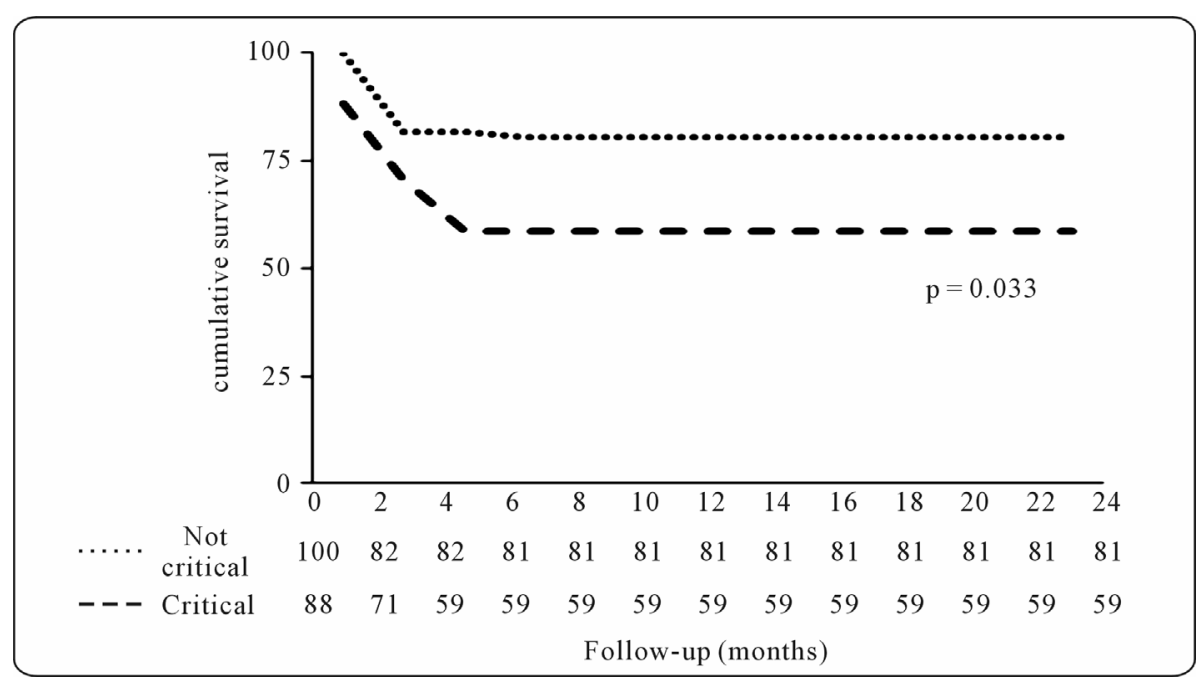

Figure 6. Overall morbidity adjusted for critical preoperative state among patients in Palestine.

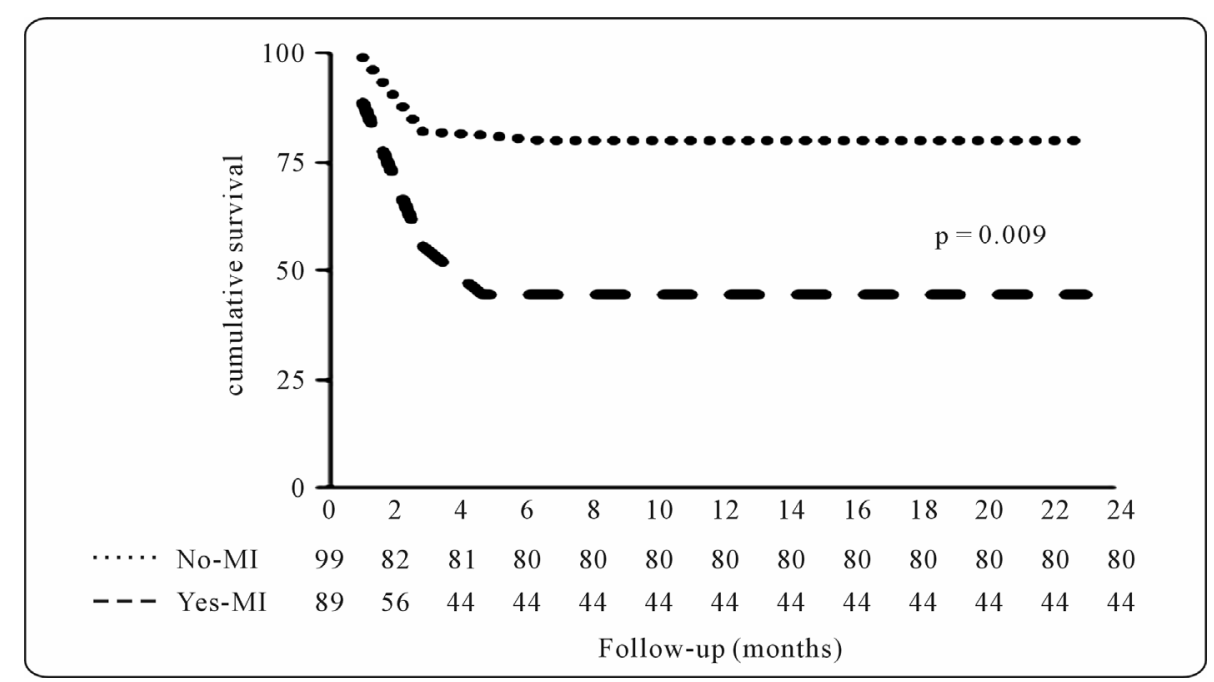

Figure 7. Overall morbidity adjusted for recent myocardial infarction (MI < 90 days) among patients in Palestine. 


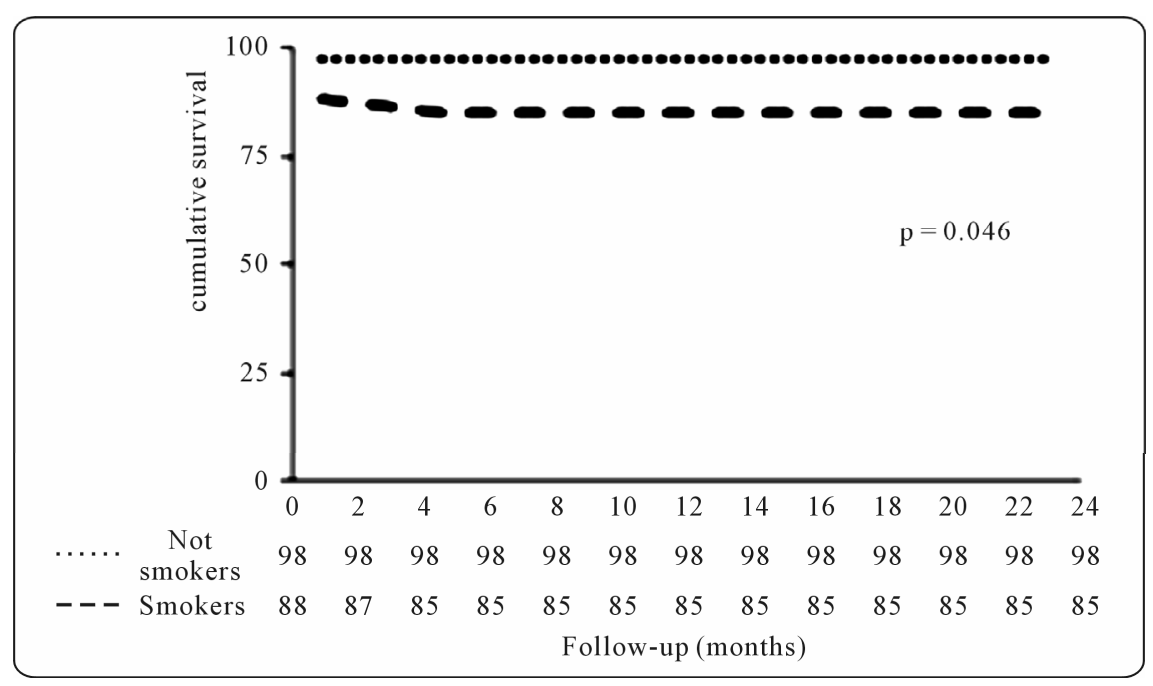

Figure 8. Overall infection adjusted for smoking among patients in Palestine.

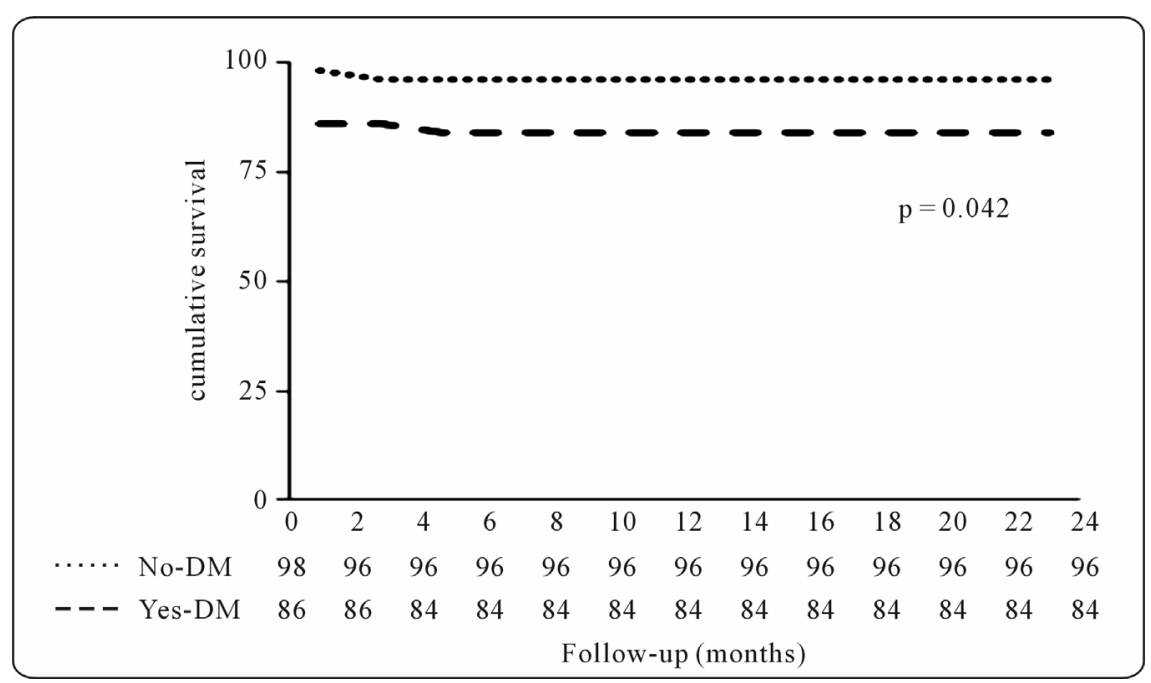

Figure 9. Overall infection adjusted for diabetes among patients in Palestine.

confounding effect of serum creatinin $>200 \mu \mathrm{mol} /$ is shown by the result of the $\log$ rank test $\mathrm{P}=0.039$ as shown in Figure 10.

\subsubsection{Atrial Fibrillation}

The two groups show significant differences in their AF morbidity experience (Figure 11). More Belgians developed this complication with differences observed in both tests (Breslow test $\mathrm{P}=0.008)$ and (log-rank test $\mathrm{P}=$ 0.008).

\subsubsection{Prediction of Hospital Readmission}

According to the univariate analysis DM $(\mathrm{P}=0.011)$ and smoking $(\mathrm{P}=0.012)$ were entered into multivariate backward logistic regression analysis and were significantly associated with higher readmission rate in Ramallah hospital (Table 7).

\subsubsection{Prediction of Mortality Using EuroSCORE}

As shown in Table 4, the mortality rate in the Palestinian group was $4 \%$, the mean standard EuroSCORE was $3.7 \pm$ 3.3 and the mean logistic EuroScore was $5.0 \pm 7.2$. In the Belgian group, the mortality was $2 \%$, the mean standard EuroSCORE was $3.9 \pm 3.3$ and the mean logistic Euro Score was $4.9 \pm 7$. According to univariate logistic regression, only the logistic EuroSCORE was an independent predictor for mortality during the follow up period in the Palestinian group, as summarized in Table 8 $(\mathrm{P}=0.013)$. Both standard and logistic euro scores were independent predictors for mortality among patients in the Belgian group ( $\mathrm{P}=0.042, \mathrm{P}=0.037$, respectively).

\subsubsection{Prediction of Hospital Stay}

The length of hospitalization in Ramallah hospital ranged from 2 - 63 days $(8.9 \pm 6.7)$. According to multivariate 


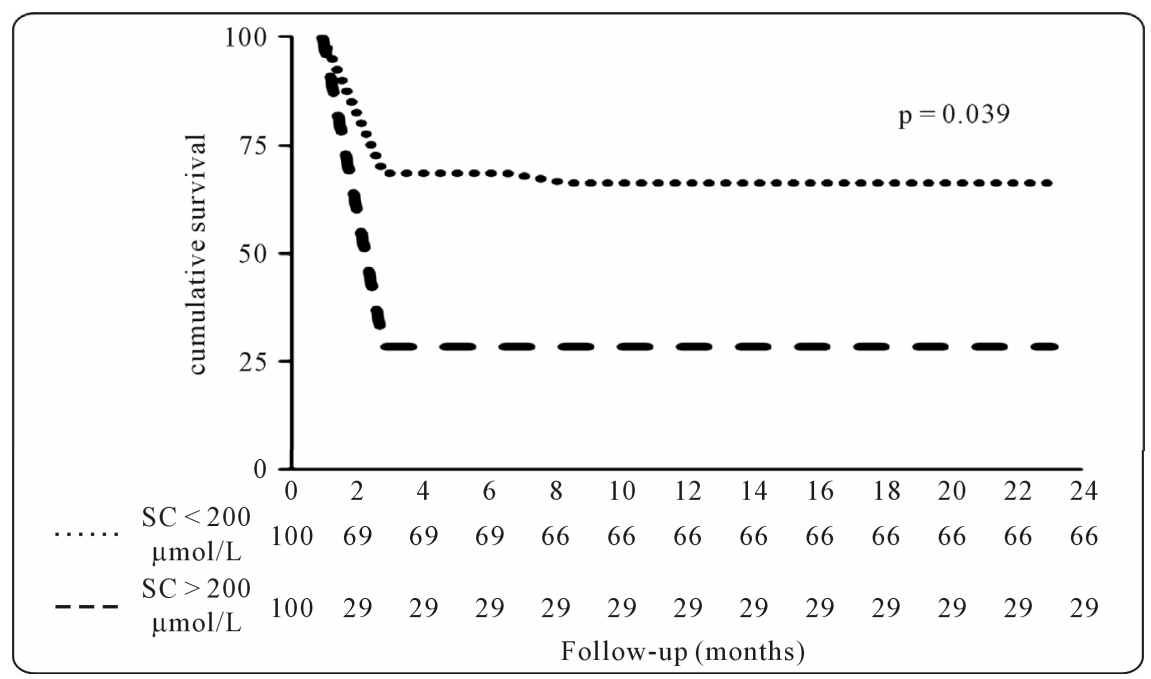

Figure 10. Overall morbidity adjusted for Serum creatinine (SC) $>200 \mu \mathrm{mol} / \mathrm{L}$ among patients in Belgium.

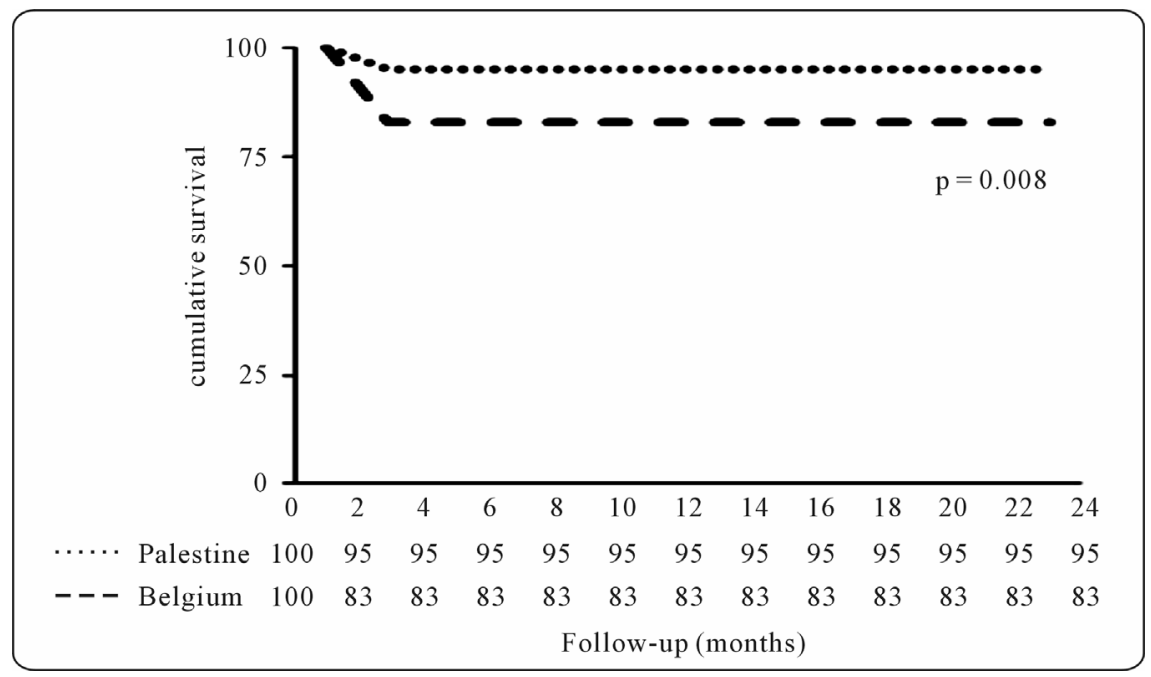

Figure 11. Atrial fibrillation among patients in the two study group.

Table 7. Predictors of total readmissions after CABAG in Ramallah hospital.

\begin{tabular}{cccc}
\hline Risk variables & B-Coefficient & p-value & Odds-Ratio \\
\hline Diabetes mellitus & 1.840 & 0.011 & 6.299 \\
Smoking & 2.716 & 0.012 & 15.114 \\
\hline
\end{tabular}

Note: Multivariate logistic model. Goodness-of-fit: Hosmer-Lemshow $\mathrm{X}^{2}=$ $104, \mathrm{df}=2(\mathrm{P}=0.950)$.

backward logistic regression summarized in Table 9, patients with risk factors of positive family history of atherosclerosis $(\mathrm{P}=0.004)$ and diabetes $(\mathrm{P}=0.017)$ were significantly associated with prolonged hospital stay. The length of hospitalization in University hospital Ghent ranged from $7-47$ days $(11.5 \pm 6.1)$. According to multivariate backward logistic regression summarized in Ta-
Table 8. Prediction of post op Mortality using EuroSCORE and Logistic-EuroSCORE.

\begin{tabular}{cccc}
\hline & B-Coefficient & P-value. & Odds-Ratio \\
\hline Palestine & & & \\
Euro-SCORE & 0.246 & 0.062 & 1.279 \\
LogisticEuroSCORE & $10 \cdot 179$ & 0.013 & 26331.187 \\
Belgium & & & \\
Euro-SCORE & 0.382 & 0.042 & 1.466 \\
LogistiEuroSCORE & 8.865 & 0.037 & 7081.799 \\
\hline
\end{tabular}

Note: Palestinian patient's Multivariate logistic model. Goodness-of-fit: Hosmer-Lemshow $\left(\mathrm{X}^{2}=9.448, \mathrm{df}=6(\mathrm{P}=0.150), \mathrm{X}^{2}=7.573, \mathrm{df}=8(\mathrm{P}=\right.$ $0.476)$ respectively and $X^{2}=5.542, \mathrm{df}=7(\mathrm{P}=0.594), \mathrm{X}^{2}=6.581, \mathrm{df}=8(\mathrm{P}$ $=0.582)$ in Belgium group. 
ble 10, patients with risk factors DM $(\mathrm{P}=0.013)$, smoking $(\mathrm{P}=0.045)$, and urgent surgery $(\mathrm{P}=0.037)$ were significantly associated with prolonged hospital stay.

\section{DISCUSSION}

Mortality post CABG serve as an indicator of the quality and effectiveness of care given by health care providers in cardiac surgery. As mortality affected by patients' preoperative characteristics, it might be incorrect to compare the quality of institutions or surgeons from different countries unless risk stratification models was implemented for the comparison which gives a good estimation of prognosis post CABG. The European System for Cardiac Operative Risk Evaluation (EuroSCORE) and the Society of Thoracic Surgeons (STS) score are the most used risk tool [15]. EuroSCORE has gained a wide acceptance and validation to predict mortality post On-pump or Off-pump CABG in different countries and institutions [16].

Our study provides first time data that presents a comparison between institutions of the European and Arab world for patients who underwent isolated CABG surgery that highlights risk factors, mortality and morbidity, length of hospitalization and readmission rates. On the other hand, there are a few studies addressing CABG in developing countries let alone in the Occupied Palestinian Territories. There were no significant differences between our groups, in terms of patient characteristics and operative data. This similarity between the two groups is quite important to guarantee that the outcomes post-CABG cannot be attributed to differences in these characteristics. In fact we encountered some difficulties to match the patients. First, we found that among Palestinian woman more and younger females undergo CABG opposite to Belgian patients. This might be explained by a higher incidence of diabetes, stress, physical inactivity, and second hand smokers [17]. A second concern, was

Table 9. Predictions of hospital stay in Ramallah hospital using backward stepwise logistic regression.

\begin{tabular}{cc}
\hline Variabel & P-value \\
\hline Diabetes mellitus & 0.017 \\
Family History & 0.004 \\
\hline
\end{tabular}

Table 10. Prediction of hospital stay in Ghent hospital using backward stepwise logistic regression.

\begin{tabular}{cc}
\hline Variabel & P-value \\
\hline Diabetes mellitus & 0.013 \\
Smoking & 0.045 \\
Urgent surgery & 0.037 \\
\hline
\end{tabular}

that in many cases only one to two coronary anastomoses were performed among Palestinians patients which is less likely to be done in Belgium where PTCA is generally recommended for single or double vessel disease. Third, we found that more urgent cases underwent CABG among Palestinian patients than Belgium patients more likely to undergo primary coronary dilatation. Finally data were easier to be accessed in Ghent University hospital because all medical records were electronic which gives more credibility.

In this study, the observed overall mortality rates in Palestinian group appeared slightly higher than the Belgium group. However, mortality rate within 30 days of the operation was significantly higher in Palestinian patients compared to the zero-mortality in the Belgium group. A recent study conducted in Al-Shifa hospital in the Gaza strip that addresses the outcomes post cardiac surgery documented a 30-day mortality rate of $6 \%$ [18]. Nevertheless, matching of the patients is of an outmost importance since Soliman Hamad and colleagues [19] showed that patients with poor ventricular function have worse outcomes and significantly higher mortality rate.

The occurrence of some in-hospital complications post-CABG was significantly higher in Ghent University hospital. The $17 \%$ occurrence of AF is reported much higher amongst patients from Belgium compared to 5\% of the Palestinian group. The occurrence of AF in Belgium was consistent with other previous studies reporting $15 \%$ to $40 \%$ of $\mathrm{AF}$ in post-CABG patients [20,21] The low incidence of AF in Ramallah hospital can be partially explained due to early use of amiodarone for all patients with sinus tachycardia. However, underreporting of post-CABG AF in Ramallah hospital cannot be excluded. The findings from Al-Shifa hospital in Gaza strip were different to those in Ramallah hospital. More serious adverse events (54\%) were reported following cardiac surgery: $18 \%$ had bleeding and 15\% infections, whereas the mortality rate within 30 days post-operation was $6 \%$ [18]. In contrast, in Ramallah hospital only $14 \%$ of the patients within 30 days of operation had severe complications: $5 \%$ had bleeding and 10\% had infections.

Different studies showed that co-morbidities including: chronic obstructive pulmonary disease (COPD), chronic renal failure (CRF), low ejection fraction and diabetes are associated with poor early outcomes and higher mortality, morbidity and re-admission rates post-CABG [2224].Variables associated with an increase in patient morbidity among Palestinian patients were critical preoperative state (17\%), unstable angina (24\%) and MI (9\%). By contrast in Belgium patients the variables at risk were CRF (7\%), COPD (11\%), and dyslipidemia (78\%). All are considered as risk factors for higher adverse events post-CABG.

An important additional finding in this study was the 
higher re-admission rate among Palestinian patients $(10 \%)$ mostly due to wound infection. This may be have been related to their high rate of DM $(50 \%)$ while the majority of patients were heavy smokers $(60 \%)$. Previous studies explained that infections post-CABG affect the patient's mortality, morbidity and length of stay. Obese patients, diabetes mellitus, and COPD are considered risk factors associated with an increase of post-surgical infections $[25,26]$.

Another confounder of the higher readmission rates amongst Palestinian patients might be the fact that their length of hospital stay was significantly lower (IQR $=6$ 10). This is partially due to the tremendous pressure of the relatives to take the patient home as soon as possible since it is the family and not the hospital that takes care of the patient's well-being while hospitalized. Another explanation is that the health services in the Palestinian territories are facing a number of challenges due to the political situation. Ramallah hospital is a referral hospital located in the center of the West-bank, due to many roadblocks and checkpoints mobility and follow up of the patients in this referral hospital is affected. These circumstances may jeopardize the safety of those patients and make the continuity of care a real problem. Henceforth the longer length of hospitalization in Belgian patients $(\mathrm{IQR}=9$ - 11$)$ could in part explain the higher occurrence of in-hospital adverse events. By contrast Cowper and colleagues found that early discharge have no effect on the risk of increasing readmission rates [27].

\section{CONCLUSION}

The results of this study revealed that during the postoperative phase, few significant differences were noticed while comparing the outcomes of patients in Palestinian and Belgian groups. Based on that, we can conclude that CABG can be done locally in Palestinian territories and can lead to similar outcomes as in Europe. Furthermore, our data supported the safety of this practice and its effectiveness for Palestinian patients. This can be explained by the presence of competent experienced multidisciplinary team who are providing quality of care for patients undergoing CABG in Palestine. Moreover, we found that some local co-morbidity can affect patient's outcomes. Diabetes and smoking in Palestine were independent risk factors for higher readmission rate and infection. The use of such results will better equip health care providers and patients in the decision making process on the indications prior CABG surgery.

\section{RECOMMENDATIONS}

There are several areas for future researches to provide additional data for CABG in Palestine. Patient satisfactions about health care systems post cardiac surgery in
Palestine is an important issue to be addressed. Researchers could also use the results of this study, as a stepping stone for further study of the link between pre/ post-surgical nursing care and post-surgical quality of life. No prior studies have addressed stress as risk factor for developing coronary heart disease which warrants further studies. Development of rehabilitation program post-CABG surgery in Ramallah hospital is necessary to provide patients and their families' with essential information about their disease, life style changes, treatment and follow-up. Finally, future research is needed to examine the reasons that lead to early $\mathrm{CABG}$ in young female patients.

\section{LIMITATIONS}

The study used a convenience selected sample for patients who underwent isolated CABG with a small number of cases in both groups which limits the generality of the study.

\section{ACKNOWLEDGEMENT}

This research has been funded by Doctoral grant from Flemish Interuniversity Council-University Development Cooperation, Belgium.

\section{REFERENCES}

[1] Cassar, A., Holmes, D.R., Rihal, C.S. and Gersh, B.J. (2009) Chronic coronary artery disease: Diagnosis and management. Mayo Clinic Proceedings, 84, 1130-1146. doi:10.4065/mcp.2009.0391

[2] Yusuf, S. (2004) Tackling the challenge of cardiovascular disease burden in developing countries. American Heart Journal, 148, 1-4. doi:10.1016/j.ahj.2004.03.045

[3] Gaziano, T.A., Bitton, A., Anand, S., Gessel, S.A. and Murphy, A. (2010) Growing epidemic of coronary heart disease in low-and middle income countries. Current Problems in Cardiology, 35, 72-115.

doi:10.1016/j.cpcardiol.2009.10.002

[4] Gersh, B.J., Sliwa, S., Mayosi, B.M. and Yusef, S. (2010) The epidemic of cardiovascular disease in the developing world: Global implications. European Heart Journal, 31, 642-648. doi:10.1093/eurheartj/ehq030

[5] Hall, S.L. and Lorenc, T. (2010) Secondary prevention of coronary artery disease. American Family Physician, 81, 289-296.

[6] Martin, C.G. and Turkelson, S.L. (2006) Nursing care of the patient undergoing coronary artery bypass grafting. Journal of Cardiovascular Nursing, 21, 109-117.

[7] Aroney, C.N., Aylward, P., Kelly, A., Chew, D.P.B., Clune, E. and Acute Coronary Syndrome Guidelines Working Group. (2006) Guidelines for the management of acute coronary syndromes. Medical Journal of Australia, 184, S1-S29.

[8] Palestinian Ministry of Health. (2012) Statistical health 
reports. http://www.moh.ps

[9] Kark, J.D., Fink, R., Adler, B., Goldberger, N. and Goldman, S. (2006) The incidence of coronary heart disease among Palestinians and Israelis in Jerusalem. International Journal of Epidemiology, 35, 448-457. doi:10.1093/ije/dy1012

[10] Jabara, R., Namouz, S., Kark, J.D. and Lotan, C. (2007) Risk characteristics of Arab and Jewish women with coronary heart disease in Jerusalem. The Israel Medical Association Journal, 9, 316-320.

[11] Ministry of Health. (2012) National strategic health plan 2011-2013. http://www.moh.ps/attach/20.pdf

[12] Nashef, S.A., Roques, F., Michel, P., Gauducheau, E., Lemeshow, S. and Salamon, R. (1999) European system for cardiac operative risk evaluation (EuroSCORE). European Journal Cardio-Thoracic Surgery, 16, 9-13. doi:10.1016/S1010-7940(99)00134-7

[13] Nashef, S.A., Roques, F., Sharples, L.D., et al. (2012) EuroSCORE II. European Journal Cardio-Thoracic Surgery, 41,734-744. doi:10.1093/ejcts/ezs043

[14] Siregar, S., Groenwold, R.H.H., De Heer, F., et al. (2012) Performance of the original. European Journal CardioThoracic Surgery, 285, 1.

[15] Ettema, R.G.A., Peelen, L.M., Schuurmans, M.J., et al. (2010) Prediction models for prolonged intensive care unit stay after cardiac surgery: Systematic Review and validation study. Circulation, 122, 682-689. doi:10.1161/CIRCULATIONAHA.109.926808

[16] Parolari, A., Pesce, L.L., Trezzi M., et al. (2009) Performance of EuroSCORE in CABG and off-pump coronary artery bypass grafting: Single institution experience and meta-analysis. European Heart Journal, 30, 297-304. doi:10.1093/eurheartj/ehn581

[17] Jabara, R., Namouz, S., Kark, J.D. and Lotan, C. (2007) Risk characteristics of Arab and Jewish women with coronary heart disease in Jerusalem. The Israel Medical Association Journal, 9, 316-320.

[18] Jamee, A. and Abed, Y. (2012) Outcomes of cardiac surgery in the Gaza Strip, occupied Palestinian territory: A cross-sectional study. Lancet Palestinian Health Alliance
Conference, 5-6 March 2012, Beirut, Lebanon, 380, S27.

[19] Hamad, M.A.S., Van Straten A.H.M., et al. (2010) Preoperative ejection fraction as a predictor of survival after coronary artery bypass grafting: Comparison with a matched general population. Journal of Cardiothoracic Surgery, 5, 29. doi:10.1186/1749-8090-5-29

[20] Mullen-Fortino, M. and O'Brien, N. (2009) Caring for a patient after coronary artery bypass graft surgery. Nursing Critical Care, 4, 22-27.

[21] Sun, X., et al. (2011) Comparison of frequency of atrial fibrillation after coronary artery bypass grafting in African Americans versus European Americans. The American Journal of Cardiology, 108, 669-672. doi:10.1016/j.amjcard.2011.04.013

[22] Robert, et al. (2002) The effect of comorbid illness on mortality outcomes in cardiac surgery. Archives of Surgery, 137,428-433. doi:10.1001/archsurg.137.4.428

[23] Mohammadi, S., et al. (2007) Long-term impact of diabetes and its comorbidities in patients undergoing isolated primary coronary artery bypass graft surgery. Circulation, 116, I102-I252.

[24] Batàr, S.C., Sarlea, S.V., Tigan, S.I. and Bàrsan, M. (2010) Gender and Diabetes Mortaliy Relevance on Outcomes of Coronary Artery Bypas Grafting. A retrospecttive study. Applied Medical Informatics, 26, 83-88.

[25] Al-Zaru, I.M., Ammouri, A.A., Al-Hassan, M.A. and Amr, A.A. (2010) Risk factors for deep sternal wound infections after cardiac surgery in Jordan. Journal of Clinical Nursing, 19, 1873-1881. doi:10.1111/j.1365-2702.2010.03193.X

[26] Ahmed, D., Chema, F.H., Ahmed, Y.I, et al. (2011) Incidence and predictors of infection in patients undergoing primary isolated coronary artery bypass grafting: A report from a tertiary care hospital in a developing country. The Journal of Cardiovascular Surgery (Torino), 52, 99-104.

[27] Cowper, P.A., et al. (2007) Is early too early? Effect of shorter stays after bypass surgery. The Annals of Thoracic Surgery, 83, 100-107. doi:10.1016/j.athoracsur.2006.08.001 